MATHEMATICS OF COMPUTATION

Volume 75, Number 255, July 2006, Pages 1217-1231

S 0025-5718(06)01859-X

Article electronically published on May 1, 2006

\title{
INTEGRAL FORMULAS FOR CHEBYSHEV POLYNOMIALS AND THE ERROR TERM \\ OF INTERPOLATORY QUADRATURE FORMULAE FOR ANALYTIC FUNCTIONS
}

\author{
SOTIRIOS E. NOTARIS
}

\begin{abstract}
We evaluate explicitly the integrals $\int_{-1}^{1} \pi_{n}(t) /(r \mp t) d t,|r| \neq 1$, with the $\pi_{n}$ being any one of the four Chebyshev polynomials of degree $n$. These integrals are subsequently used in order to obtain error bounds for interpolatory quadrature formulae with Chebyshev abscissae, when the function to be integrated is analytic in a domain containing $[-1,1]$ in its interior.
\end{abstract}

\section{INTRODUCTION}

The usefulness of Chebyshev polynomials in numerical analysis is undisputed. This is largely due to their nice properties, many of which follow from the trigonometric representations these polynomials satisfy on the interval $[-1,1]$. An immediate consequence of these representations is that the zeros of Chebyshev polynomials can be given by explicit formulas, and this makes them attractive choices for nodes of interpolatory quadrature formulae. Notable examples of the latter are the wellknown Fejér and Clenshaw-Curtis rules.

In the following, we enlarge the list of properties of Chebyshev polynomials by showing that the integrals

$$
\int_{-1}^{1} \frac{\pi_{n}(t)}{r \mp t} d t, \quad|r| \neq 1,
$$

with the $\pi_{n}$ being any one of the four Chebyshev polynomials of degree $n$, can be computed explicitly. This finds immediate application in estimating the error of certain interpolatory formulae with Chebyshev abscissae, by means of Hilbert space or contour integration methods, when the function to be integrated is analytic in a domain containing $[-1,1]$ in its interior. The quality of these estimates, and a comparison with already existing ones, is illustrated with a numerical example.

Received by the editor May 13, 2004 and, in revised form, October 3, 2004.

2000 Mathematics Subject Classification. Primary 33C45, 65D32.

Key words and phrases. Integral formulas, Chebyshev polynomials, interpolatory quadrature formulae, error bounds.

This work was supported in part by a grant from the Research Committee of the University of Athens, Greece, and in part by a "Pythagoras" O.P. Education grant to the University of Athens from the Ministry of National Education, Greece, and the European Union.

(C)2006 American Mathematical Society Reverts to public domain 28 years from publication 


\section{Integral FORMulas FOR Chebyshev polynomials}

The $n$th degree Chebyshev polynomials $T_{n}, U_{n}, V_{n}$, and $W_{n}$ of the first, second, third, and fourth kind, respectively, are characterized by the well-known representations

$$
\begin{gathered}
T_{n}(\cos \theta)=\cos n \theta, \quad U_{n}(\cos \theta)=\frac{\sin (n+1) \theta}{\sin \theta}, \\
V_{n}(\cos \theta)=\frac{\cos (n+1 / 2) \theta}{\cos (\theta / 2)}, \quad W_{n}(\cos \theta)=\frac{\sin (n+1 / 2) \theta}{\sin (\theta / 2)} .
\end{gathered}
$$

We first give some simple relations among these polynomials, which will be useful in the subsequent development.

Lemma 2.1. Let $U_{-1}(t)=0$. Then

$$
\begin{aligned}
T_{n}(t) & =\frac{1}{2}\left\{U_{n}(t)-U_{n-2}(t)\right\}, & & n=1,2, \ldots, \\
V_{n}(t) & =U_{n}(t)-U_{n-1}(t), & & n=0,1,2, \ldots, \\
W_{n}(t) & =U_{n}(t)+U_{n-1}(t), & n & =0,1,2, \ldots .
\end{aligned}
$$

Proof. Formulas (2.3) and (2.4) follow from the corresponding formulas for the difference of sines

$$
\begin{gathered}
\sin (n+1) \theta-\sin (n-1) \theta=2 \cos n \theta \sin \theta, \\
\sin (n+1) \theta-\sin n \theta=2 \cos (n+1 / 2) \theta \sin (\theta / 2),
\end{gathered}
$$

and (2.1)-(2.2)

Also, replacing $\theta$ by $\pi+\theta$ in the second equation in (2.1) and (2.2) gives

$$
\begin{array}{ll}
U_{n}(t)=(-1)^{n} U_{n}(-t), & n=0,1,2, \ldots, \\
W_{n}(t)=(-1)^{n} V_{n}(-t), & n=0,1,2, \ldots,
\end{array}
$$

which, together with (2.4), implies (2.5).

We can now present the announced explicit forms for the integrals (1.1). The pattern was discovered by computing, analytically, enough of these integrals for the first few values of $n$. We examine separately the cases $|r|>1$ and $|r|<1$.

Proposition 2.2. Let $r \in \mathbb{R}$ with $|r|>1$.

(i) If $\pi_{n}=T_{n}$, then

$$
\int_{-1}^{1} \frac{\pi_{n}(t)}{r-t} d t=\pi_{n}(r) \ln \left(\frac{r+1}{r-1}\right)-4 \sum_{k=1}^{[(n+1) / 2]} \frac{\pi_{n-2 k+1}(r)}{2 k-1},
$$

while, if $\pi_{n}=U_{n}, \pi_{n}=V_{n}$, or $\pi_{n}=W_{n}$, then

$$
\int_{-1}^{1} \frac{\pi_{n}(t)}{r-t} d t=\pi_{n}(r) \ln \left(\frac{r+1}{r-1}\right)-4 \sum_{k=1}^{[(n+1) / 2]} \frac{\pi_{n-2 k+1}(r)}{2 k-1} .
$$

(ii) If $\pi_{n}=T_{n}$, then

$$
\int_{-1}^{1} \frac{\pi_{n}(t)}{r+t} d t=\pi_{n}(-r) \ln \left(\frac{r+1}{r-1}\right)+4 \sum_{k=1}^{[(n+1) / 2]} \frac{\pi_{n-2 k+1}(-r)}{2 k-1},
$$


while, if $\pi_{n}=U_{n}, \pi_{n}=V_{n}$, or $\pi_{n}=W_{n}$, then

$$
\int_{-1}^{1} \frac{\pi_{n}(t)}{r+t} d t=\pi_{n}(-r) \ln \left(\frac{r+1}{r-1}\right)+4 \sum_{k=1}^{[(n+1) / 2]} \frac{\pi_{n-2 k+1}(-r)}{2 k-1} .
$$

By [.] we denote the integer part of a real number, while the notation $\sum^{\prime}$ means that the last term in the sum must be halved when $n$ is odd.

Proof. (i) We first prove (2.9) with $\pi_{n}=U_{n}$. To this end, we apply induction on $n$. For $n=1$, an easy computation, using the second equation in (2.1), gives

$$
\int_{-1}^{1} \frac{U_{1}(t)}{r-t} d t=U_{1}(r) \ln \left(\frac{r+1}{r-1}\right)-4 .
$$

Assume now that the formula is true for the indices $n-1$ and $n$, and we want to prove it for the index $n+1$. It is well known that the Chebyshev polynomials of the second kind satisfy the three-term recurrence relation

$$
\begin{gathered}
U_{m+1}(t)=2 t U_{m}(t)-U_{m-1}(t), \quad m=0,1,2, \ldots, \\
U_{-1}(t)=0, \quad U_{0}(t)=1 .
\end{gathered}
$$

Applying (2.12) with $m=n$, dividing by $r-t$ and taking the integral on both sides, we have

$$
\int_{-1}^{1} \frac{U_{n+1}(t)}{r-t} d t=2 \int_{-1}^{1} \frac{t U_{n}(t)}{r-t} d t-\int_{-1}^{1} \frac{U_{n-1}(t)}{r-t} d t
$$

Furthermore, adding and subtracting the term $r U_{n}(t)$ in the numerator of the first integral on the right side of (2.13), we get

$$
\int_{-1}^{1} \frac{U_{n+1}(t)}{r-t} d t=-2 \int_{-1}^{1} U_{n}(t) d t+2 r \int_{-1}^{1} \frac{U_{n}(t)}{r-t} d t-\int_{-1}^{1} \frac{U_{n-1}(t)}{r-t} d t .
$$

We now consider two cases for $n$. When $n$ is even, (2.14), by virtue of the induction hypothesis and

yields

$$
\int_{-1}^{1} U_{m}(t) d t= \begin{cases}\frac{2}{m+1} & \text { if } m \text { is even } \\ 0 & \text { if } m \text { is odd }\end{cases}
$$

$$
\begin{aligned}
& \int_{-1}^{1} \frac{U_{n+1}(t)}{r-t} d t=\left\{2 r U_{n}(r)-U_{n-1}(r)\right\} \ln \left(\frac{r+1}{r-1}\right) \\
&-4\left\{\left\{2 r U_{n-1}(r)-U_{n-2}(r)\right\}+\frac{1}{3}\left\{2 r U_{n-3}(r)-U_{n-4}(r)\right\}\right. \\
&\left.+\cdots+\frac{1}{n-1}\left\{2 r U_{1}(r)-U_{0}(r)\right\}+\frac{1}{n+1}\right\}
\end{aligned}
$$

which, in view of (2.12), gives

$$
\begin{aligned}
\int_{-1}^{1} \frac{U_{n+1}(t)}{r-t} d t= & U_{n+1}(r) \ln \left(\frac{r+1}{r-1}\right) \\
& -4\left\{U_{n}(r)+\frac{1}{3} U_{n-2}(r)+\cdots+\frac{1}{n-1} U_{2}(r)+\frac{1}{n+1}\right\}
\end{aligned}
$$


Also, for $n$ odd, we obtain in a like manner,

$$
\begin{aligned}
\int_{-1}^{1} \frac{U_{n+1}(t)}{r-t} d t= & U_{n+1}(r) \ln \left(\frac{r+1}{r-1}\right) \\
& -4\left\{U_{n}(r)+\frac{1}{3} U_{n-2}(r)+\cdots+\frac{1}{n-2} U_{3}(r)+\frac{1}{n} U_{1}(r)\right\},
\end{aligned}
$$

which, together with (2.15), combines to

$$
\int_{-1}^{1} \frac{U_{n+1}(t)}{r-t} d t=U_{n+1}(r) \ln \left(\frac{r+1}{r-1}\right)-4 \sum_{k=1}^{[((n+1)+1) / 2]} \frac{U_{n+1-2 k+1}(r)}{2 k-1},
$$

proving the induction claim, and concluding the induction.

Also, from (2.3),

$$
\int_{-1}^{1} \frac{T_{n}(t)}{r-t} d t=\frac{1}{2}\left\{\int_{-1}^{1} \frac{U_{n}(t)}{r-t} d t-\int_{-1}^{1} \frac{U_{n-2}(t)}{r-t} d t\right\},
$$

and inserting (2.9) with $\pi_{n}=U_{n}$ and $\pi_{n-2}=U_{n-2}$, we find, taking into account that $U_{-1}(t)=0$,

$$
\begin{aligned}
\int_{-1}^{1} \frac{T_{n}(t)}{r-t} d t & \\
= & \frac{1}{2}\left\{U_{n}(r)-U_{n-2}(r)\right\} \ln \left(\frac{r+1}{r-1}\right) \\
& -4\left\{\frac{1}{2}\left\{U_{n-1}(r)-U_{n-3}(r)\right\}+\frac{1}{3} \frac{1}{2}\left\{U_{n-3}(r)-U_{n-5}(r)\right\}\right. \\
& +\cdots+\left\{\begin{array}{ll}
\frac{1}{n-3} \frac{1}{2}\left\{U_{3}(r)-U_{1}(r)\right\}+\frac{1}{n-1} \frac{1}{2}\left\{U_{1}(r)-U_{-1}(r)\right\} & \text { if } n \text { is even } \\
\frac{1}{n-2} \frac{1}{2}\left\{U_{2}(r)-U_{0}(r)\right\}+\frac{1}{2} \frac{1}{n} & \text { if } n \text { is odd }
\end{array}\right\},
\end{aligned}
$$

which, by (2.3), shows (2.8).

The proof of (2.9) with $\pi_{n}=V_{n}$ or $\pi_{n}=W_{n}$ is similar to that of (2.8), if we use (2.4) and (2.5) in place of (2.3).

(ii) Replacing $\theta$ by $\pi+\theta$ in (2.1) and (2.2) gives

$$
\begin{array}{cl}
T_{n}(t) & =(-1)^{n} T_{n}(-t), \quad n=0,1,2, \ldots, \\
V_{n}(t) & =(-1)^{n} W_{n}(-t), \quad n=0,1,2, \ldots,
\end{array}
$$

which, inserted, together with (2.6) and (2.7), into (2.8) and (2.9), and changing variables from $-t$ to $t$ in the integrals involved there, yield (2.10) and (2.11).

We now turn to the integrals (1.1) with $|r|<1$, which, in view of the singularity at $r$ or $-r$, will be computed in the Cauchy principal value sense.

We recall that if $p$ is an interior point of an interval $[a, b]$ in $\mathbb{R}$ and $f$ is a function defined at every point of $[a, b]$ except perhaps $p$, then the Cauchy principal value of the integral of $f$ over $[a, b]$, denoted by $f_{a}^{b} f(t) d t$, is given by

$$
f_{a}^{b} f(t) d t=\lim _{\epsilon \rightarrow 0^{+}}\left\{\int_{a}^{p-\epsilon} f(t) d t+\int_{p+\epsilon}^{b} f(t) d t\right\}
$$


(cf. [2, Section 32]). An interesting case of a Cauchy principal value integral is the so-called Hilbert transform of a function $f$,

$$
f_{a}^{b} \frac{f(t)}{x-t} d t, \quad x \in(a, b)
$$

(cf. [4, Section 1.6]). The integrals (1.1) with $|r|<1$ are precisely of this type.

Proposition 2.3. Let $r \in \mathbb{R}$ with $|r|<1$.

(i) If $\pi_{n}=T_{n}$, then

$$
f_{-1}^{1} \frac{\pi_{n}(t)}{r-t} d t=\pi_{n}(r) \ln \left(\frac{1+r}{1-r}\right)-4 \sum_{k=1}^{[(n+1) / 2]} \frac{\pi_{n-2 k+1}(r)}{2 k-1}
$$

while, if $\pi_{n}=U_{n}, \pi_{n}=V_{n}$, or $\pi_{n}=W_{n}$, then

$$
f_{-1}^{1} \frac{\pi_{n}(t)}{r-t} d t=\pi_{n}(r) \ln \left(\frac{1+r}{1-r}\right)-4 \sum_{k=1}^{[(n+1) / 2]} \frac{\pi_{n-2 k+1}(r)}{2 k-1} .
$$

(ii) If $\pi_{n}=T_{n}$, then

$$
f_{-1}^{1} \frac{\pi_{n}(t)}{r+t} d t=\pi_{n}(-r) \ln \left(\frac{1+r}{1-r}\right)+4 \sum_{k=1}^{[(n+1) / 2]} \frac{\pi_{n-2 k+1}(-r)}{2 k-1}
$$

while, if $\pi_{n}=U_{n}, \pi_{n}=V_{n}$, or $\pi_{n}=W_{n}$, then

$$
f_{-1}^{1} \frac{\pi_{n}(t)}{r+t} d t=\pi_{n}(-r) \ln \left(\frac{1+r}{1-r}\right)+4 \sum_{k=1}^{[(n+1) / 2]} \frac{\pi_{n-2 k+1}(-r)}{2 k-1} .
$$

$B y[\cdot]$ we denote the integer part of a real number, while the notation $\sum^{\prime}$ means that the last term in the sum must be halved when $n$ is odd.

Proof. The proof follows the steps of the proof in Proposition 2.2.

(i) We first prove (2.18) with $\pi_{n}=U_{n}$ by applying induction on $n$. For $n=1$, utilizing (2.16), we find

$$
f_{-1}^{1} \frac{U_{1}(t)}{r-t} d t=U_{1}(r) \ln \left(\frac{1+r}{1-r}\right)-4
$$

Then, assuming that (2.18) is true for the indices $n-1$ and $n$, and proceeding precisely as in the case $|r|>1$, we show that it is also true for the index $n+1$, concluding that way the induction.

The proof of (2.17) and (2.18) with $\pi_{n}=V_{n}$ or $\pi_{n}=W_{n}$ is almost identical to the proof of the corresponding cases in Proposition 2.2 .

(ii) Formulas (2.19) and (2.20) can be derived most easily if we note that

$$
f_{-1}^{1} \frac{\pi_{n}(t)}{r+t} d t=-f_{-1}^{1} \frac{\pi_{n}(t)}{(-r)-t} d t
$$

and then apply formulas (2.17) and (2.18), respectively, with $r$ replaced by $-r$. 


\section{ERror Bounds For INTERPOLATORY QUADRATURE FORMUlAE}

An interpolatory quadrature formula for approximating the integral $\int_{-1}^{1} f(t) d t$ based on the $n$ distinct points $\tau_{1}, \tau_{2}, \ldots, \tau_{n}$, ordered decreasingly, in the interval $(-1,1)$ is that constructed by integrating the inteprolating polynomial $p_{n-1}\left(f ; \tau_{1}, \tau_{2}, \ldots, \tau_{n} ; t\right)$, which leads to

$$
\int_{-1}^{1} f(t) d t=\sum_{\nu=1}^{n} w_{\nu} f\left(\tau_{\nu}\right)+R_{n}(f) .
$$

By definition (3.1) has degree of exactness at least $n-1$; i.e., $R_{n}(f)=0$ for all $f \in \mathbb{P}_{n-1}$.

One way to obtain an estimate for the error term of (3.1) is by using a Hilbert space method proposed by Hämmerlin in [6]. If $f$ is a single-valued holomorphic function in the $\operatorname{disc} C_{r}=\{z \in \mathbb{C}:|z|<r\}, r>1$, then it can be written as

$$
f(z)=\sum_{k=0}^{\infty} a_{k} z^{k}, \quad z \in C_{r} .
$$

Define

$$
|f|_{r}=\sup \left\{\left|a_{k}\right| r^{k}: k \in \mathbb{N}_{0} \text { and } R_{n}\left(t^{k}\right) \neq 0\right\},
$$

which is a seminorm in the space

$$
X_{r}=\left\{f: f \text { holomorphic in } C_{r} \text { and }|f|_{r}<\infty\right\} .
$$

Then the error term $R_{n}$ in (3.1) is a continuous linear functional in $\left(X_{r},|\cdot|_{r}\right)$, and its error norm is given by

$$
\left\|R_{n}\right\|=\sum_{k=d+1}^{\infty} \frac{\left|R_{n}\left(t^{k}\right)\right|}{r^{k}}
$$

where $d$ is precise degree of exactness of (3.1) (cf. 11, Section 1.1]). If, in addition,

$$
R_{n}\left(t^{k}\right) \geq 0, \quad k=0,1,2, \ldots,
$$

or

$$
(-1)^{k} R_{n}\left(t^{k}\right) \geq 0, \quad k=0,1,2, \ldots,
$$

then, letting

$$
\pi_{n}(t)=\prod_{\nu=1}^{n}\left(t-\tau_{\nu}\right)
$$

we can derive the representations

$$
\left\|R_{n}\right\|=\frac{r}{\pi_{n}(r)} \int_{-1}^{1} \frac{\pi_{n}(t)}{r-t} d t
$$

or

$$
\left\|R_{n}\right\|=\frac{r}{\pi_{n}(-r)} \int_{-1}^{1} \frac{\pi_{n}(t)}{r+t} d t,
$$

respectively (see [1, Section 1.2]). Consequently, if $f \in X_{R}$,

$$
\left|R_{n}(f)\right| \leq\left\|R_{n}\right\||f|_{r}, \quad 1<r \leq R
$$


and optimizing the bound on the right of (3.5) as a function of $r$, we get

$$
\left|R_{n}(f)\right| \leq \inf _{1<r \leq R}\left(\left\|R_{n}\right\||f|_{r}\right)
$$

An alternate way to obtain an estimate for the error term of (3.1) is by using a contour integration method. If $f$ is a single-valued holomorphic function in a domain $D$ containing $[-1,1]$ in its interior, and $\bar{C}_{r}=\{z \in \mathbb{C}:|z|=r\}, r>1$, is a contour in $D$ surrounding $[-1,1]$, then the $R_{n}(\cdot)$ can be represented as

$$
R_{n}(f)=\frac{1}{2 \pi i} \int_{\bar{C}_{r}} K_{n}(z) f(z) d z
$$

where the kernel $K_{n}$ is given by

$$
K_{n}(z)=R_{n}\left(\frac{1}{z-\cdot}\right)=\frac{1}{\pi_{n}(z)} \int_{-1}^{1} \frac{\pi_{n}(t)}{z-t} d t,
$$

with

$$
\pi_{n}(z)=\prod_{\nu=1}^{n}\left(z-\tau_{\nu}\right)
$$

From (3.7), there immediately follows

$$
\left|R_{n}(f)\right| \leq r \max _{z \in \bar{C}_{r}}\left|K_{n}(z)\right| \max _{z \in \bar{C}_{r}}|f(z)|
$$

Now,

$$
\max _{z \in \bar{C}_{r}}\left|K_{n}(z)\right|= \begin{cases}K_{n}(r) & \text { if } R_{n} \text { satisfies }\left(3.3_{i}\right), \\ \left|K_{n}(-r)\right| & \text { if } R_{n} \text { satisfies }\left(3.3_{i i}\right)\end{cases}
$$

(see [5, Sections 2, 3, and 4]), hence, in this case, (3.9) gives, in view of (3.8), (3.10) and $\left(3.4_{i}\right)-\left(3.4_{i i}\right)$,

$$
\left|R_{n}(f)\right| \leq\left\|R_{n}\right\| \max _{|z|=r}|f(z)|
$$

with the $\left\|R_{n}\right\|$ given by $\left(3.4_{i}\right)$ or $\left(3.4_{i i}\right)$ accordingly as $R_{n}$ satisfies $\left(3.3_{i}\right)$ or $\left(3.3_{i i}\right)$, respectively. Incidentally, (3.11) can also be derived from (3.5), if $|f|_{r}$ is estimated by $\max _{|z|=r}|f(z)|$, which, for $f \in X_{R}$, exists at least for $r<R$. Consequently, the bound on the right of (3.11) can be optimized as a function of $r$ giving

$$
\left|R_{n}(f)\right| \leq \inf _{1<r<R}\left(\left\|R_{n}\right\| \max _{|z|=r}|f(z)|\right) .
$$

Among all interpolatory formulae, of particular interest are those based on the zeros of Chebyshev polynomials of any one of the four kinds, primarily, because their nodes and weights can be expressed in explicit form. Formulae of this kind were introduced by Fejér in 1933; however, their practical importance was shown by Clenshaw and Curtis in 1960 who used them in automatic computing. A detailed description of all interpolatory formulae with Chebyshev abscissae is given in [7].

Here we consider formulae of type (3.1) with the $\tau_{\nu}$ being zeros of the $n$th degree Chebyshev polynomial of the second, third, or fourth kind. The nodes and weights 
of these formulae are given by

$$
\begin{aligned}
\tau_{\nu}^{(2)}=\cos \theta_{\nu}^{(2)}, & \theta_{\nu}^{(2)}=\frac{\nu}{n+1} \pi, & \nu=1,2, \ldots, n, \\
\tau_{\nu}^{(3)}=\cos \theta_{\nu}^{(3)}, & \theta_{\nu}^{(3)}=\frac{2 \nu-1}{2 n+1} \pi, & \nu=1,2, \ldots, n, \\
\tau_{\nu}^{(4)}=\cos \theta_{\nu}^{(4)}, & \theta_{\nu}^{(4)}=\frac{2 \nu}{2 n+1} \pi, & \nu=1,2, \ldots, n
\end{aligned}
$$

(see [7, Equations (2.4)-(2.6)]), and

$$
\begin{array}{r}
w_{\nu}^{(i)}=\frac{2}{n+\alpha}\left\{1-2 \sum_{k=1}^{[(n-1) / 2]} \frac{\cos 2 k \theta_{\nu}^{(i)}}{4 k^{2}-1}-\frac{\cos 2[(n+1) / 2] \theta_{\nu}^{(i)}}{2[(n+1) / 2]-1}\right\}, \\
\nu=1,2, \ldots, n, \quad i=2,3,4,
\end{array}
$$

or, alternatively,

$$
w_{\nu}^{(i)}=\frac{4 \sin \theta_{\nu}^{(i)}}{n+\alpha} \sum_{k=1}^{[(n+1) / 2]} \frac{\sin (2 k-1) \theta_{\nu}^{(i)}}{2 k-1}, \quad \nu=1,2, \ldots, n, \quad i=2,3,4,
$$

where

$$
\alpha= \begin{cases}1 & \text { if } i=2, \\ 1 / 2 & \text { if } i=3,4,\end{cases}
$$

and $[\cdot]$ denotes the integer part of a real number. Furthermore, the $w_{\nu}^{(i)}, \nu=$ $1,2, \ldots, n, i=2,3,4$, are all positive (see [7, Section 2.1]).

Formula (3.1) with $\tau_{\nu}=\tau_{\nu}^{(2)}$, known as the Fejér rule of the second kind or the Filippi rule, has precise degree of exactness $2[(n+1) / 2]-1$ (cf. [7, Section 2.2]), and it is positive definite, satisfying

$$
R_{n}^{(2)}(f)=\frac{1}{2^{n-1}(n+1)} \frac{f^{(n)}\left(\xi_{e}\right)}{n !}, \quad \xi_{e} \in[-1,1],
$$

for $n$ even and $f \in C^{n}[-1,1]$, and

$$
R_{n}^{(2)}(f)=\frac{n+1}{2^{n-1} n(n+2)} \frac{f^{(n+1)}\left(\xi_{o}\right)}{(n+1) !}, \quad \xi_{o} \in[-1,1],
$$

for $n$ odd and $f \in C^{n+1}[-1,1]$ (see $\left[1\right.$, Section 1.7]). From $\left(3.16_{e}\right)$ and $\left(3.16_{o}\right)$, one can immediately deduce that

$$
R_{n}^{(2)}\left(t^{2 k}\right) \geq 0 \quad \text { for all } k \geq[(n+1) / 2],
$$

while, by symmetry,

$$
R_{n}^{(2)}\left(t^{2 k+1}\right)=0 \quad \text { for all } k \geq 0 .
$$

On the other hand, formula (3.1) with $\tau_{\nu}=\tau_{\nu}^{(3)}$ or $\tau_{\nu}=\tau_{\nu}^{(4)}$ has precise degree of exactness $n-1$, and it is nondefinite (cf. [7, Sections 2.2 and 2.4]). However, as the next lemma shows, the error term of this formula follows a pattern analogous to that of $\left(3.17_{e}\right)$.

Lemma 3.1. Consider the interpolatory quadrature formula (3.1).

(i) If $\tau_{\nu}=\tau_{\nu}^{(3)}$, then the error term $R_{n}=R_{n}^{(3)}$ satisfies

$$
(-1)^{k} R_{n}^{(3)}\left(t^{k}\right)>0 \quad \text { for all } k \geq K_{n} .
$$


(ii) If $\tau_{\nu}=\tau_{\nu}^{(4)}$, then the error term $R_{n}=R_{n}^{(4)}$ satisfies

$$
R_{n}^{(4)}\left(t^{k}\right)>0 \quad \text { for all } k \geq K_{n}
$$

In both cases, $K_{n}$ is a constant, $K_{n} \geq n$.

Proof. (i) Assume first that $k=2 l$ is even. From (3.1), taking into account that $w_{\nu}=w_{\nu}^{(3)}>0, \nu=1,2, \ldots, n$, we get

$$
R_{n}^{(3)}\left(t^{2 l}\right)=\sum_{\nu=1}^{n}\left\{\frac{2}{n(2 l+1)}-w_{\nu}^{(3)}\left(\tau_{\nu}^{(3)}\right)^{2 l}\right\} .
$$

Since $0<\left|\tau_{\nu}^{(3)}\right|<1, \nu=1,2, \ldots, n$, we have

$$
\lim _{l \rightarrow \infty}(2 l+1)\left(\tau_{\nu}^{(3)}\right)^{2 l}=0, \quad \nu=1,2, \ldots, n,
$$

which implies

$$
\frac{2}{n(2 l+1)}-w_{\nu}^{(3)}\left(\tau_{\nu}^{(3)}\right)^{2 l}>0 \quad \text { for all } l \geq K_{n, \nu}^{e}, \quad \nu=1,2, \ldots, n .
$$

Now, taking $K_{n}^{e}=\max _{1 \leq \nu \leq n}\left\{K_{n, \nu}^{e}\right\}$, we obtain, from (3.20), in view of (3.21),

$$
R_{n}^{(3)}\left(t^{2 l}\right)>0 \text { for all } l \geq K_{n}^{e} .
$$

If, on the other hand, $k=2 l+1$ is odd, then (3.1) gives

$$
R_{n}^{(3)}\left(t^{2 l+1}\right)=-\sum_{\nu=1}^{n} w_{\nu}^{(3)}\left(\tau_{\nu}^{(3)}\right)^{2 l+1} .
$$

We consider two cases for $n$. First, $n=2 m$ is even. Then (3.23) takes the form

$$
\begin{aligned}
R_{n}^{(3)}\left(t^{2 l+1}\right) & =-\sum_{\nu=1}^{m}\left\{w_{\nu}^{(3)}\left(\tau_{\nu}^{(3)}\right)^{2 l+1}+w_{n-\nu+1}^{(3)}\left(\tau_{n-\nu+1}^{(3)}\right)^{2 l+1}\right\} \\
& =-\sum_{\nu=1}^{m} w_{\nu}^{(3)}\left(\tau_{\nu}^{(3)}\right)^{2 l+1}\left\{1-\frac{w_{n-\nu+1}^{(3)}}{w_{\nu}^{(3)}}\left(\frac{-\tau_{n-\nu+1}^{(3)}}{\tau_{\nu}^{(3)}}\right)^{2 l+1}\right\},
\end{aligned}
$$

with the $\tau_{\nu}^{(3)}$ satisfying

$$
\begin{gathered}
\tau_{\nu}^{(3)}>0, \quad \nu=1,2, \ldots, m, \\
\tau_{n-\nu+1}^{(3)}<0, \quad \nu=1,2, \ldots, m, \\
\left|\tau_{\nu}^{(3)}\right|>\left|\tau_{n-\nu+1}^{(3)}\right|, \quad \nu=1,2, \ldots, m .
\end{gathered}
$$

Hence,

implying

$$
\lim _{l \rightarrow \infty}\left(\frac{-\tau_{n-\nu+1}^{(3)}}{\tau_{\nu}^{(3)}}\right)^{2 l+1}=0, \quad \nu=1,2, \ldots, m
$$

$$
1-\frac{w_{n-\nu+1}^{(3)}}{w_{\nu}^{(3)}}\left(\frac{-\tau_{n-\nu+1}^{(3)}}{\tau_{\nu}^{(3)}}\right)^{2 l+1}>0 \quad \text { for all } l \geq K_{n, \nu}^{o}, \quad \nu=1,2, \ldots, m,
$$

and taking $K_{n}^{o}=\max _{1 \leq \nu \leq m}\left\{K_{n, \nu}^{o}\right\}$, we obtain, from (3.24), in view of the positivity of $w_{\nu}^{(3)},(3.25)$, and (3.28),

$$
R_{n}^{(3)}\left(t^{2 l+1}\right)<0 \quad \text { for all } l \geq K_{n}^{o} .
$$


TABLE 3.1. Values of $K_{n}, 1 \leq n \leq 20$

\begin{tabular}{cccc}
\hline$n$ & $K_{n}$ & $n$ & $K_{n}$ \\
\hline 1 & 2 & 11 & 480 \\
2 & 8 & 12 & 572 \\
3 & 28 & 13 & 692 \\
4 & 48 & 33 & 804 \\
5 & 84 & 15 & 948 \\
6 & 122 & 16 & 1080 \\
7 & 178 & 17 & 1246 \\
8 & 232 & 18 & 1398 \\
9 & 308 & 19 & 1590 \\
10 & 382 & 20 & 1764 \\
\hline
\end{tabular}

If, on the other hand, $n=2 m+1$ is odd, then (3.1) gives

$$
R_{n}^{(3)}\left(t^{2 l+1}\right)=-\sum_{\nu=1}^{m}\left\{w_{\nu}^{(3)}\left(\tau_{\nu}^{(3)}\right)^{2 l+1}+w_{n-\nu+1}^{(3)}\left(\tau_{n-\nu+1}^{(3)}\right)^{2 l+1}\right\}-w_{m+1}^{(3)}\left(\tau_{m+1}^{(3)}\right)^{2 l+1},
$$

with the $\tau_{\nu}^{(3)}$ satisfying (3.25)-(3.27) and in addition $\tau_{m+1}^{(3)}>0$, thus, in a like manner as in the case of $n$ even, we get (3.29).

Now, taking $K_{n}=\max \left\{2 K_{n}^{e}, 2 K_{n}^{o}+1\right\},(3.22)$ and (3.29) are merged to (3.18).

(ii) Replacing $\nu$ by $n-\nu+1$ in (3.14), and using (3.15), gives

$$
\tau_{\nu}^{(4)}=-\tau_{n-\nu+1}^{(3)}, \quad \nu=1,2, \ldots, n,
$$

which, inserted into (3.1), yields

$$
\begin{gathered}
w_{\nu}^{(4)}=w_{n-\nu+1}^{(3)}, \quad \nu=1,2, \ldots, n, \\
R_{n}^{(4)}(f(\cdot))=R_{n}^{(3)}(f(-\cdot)) .
\end{gathered}
$$

The latter, together with (3.18), implies (3.19).

The proof of Lemma 3.1 presents a method for computing the constants $K_{n}$ in (3.18)-(3.19). Their values for $1 \leq n \leq 20$ are shown in Table 3.1.

Also, having examined (3.18)-(3.19) for the remaining values of $k$, we have found that, for $1 \leq n \leq 20$, both of them hold true when $n \leq k \leq K_{n}-1$. So, summarizing

$$
\begin{gathered}
(-1)^{k} R_{n}^{(3)}\left(t^{k}\right)>0 \text { for all } k \geq n, 1 \leq n \leq 20, \\
R_{n}^{(4)}\left(t^{k}\right)>0 \quad \text { for all } k \geq n, 1 \leq n \leq 20 .
\end{gathered}
$$

In addition, our numerical results suggest the following.

Conjecture 3.2. Consider the interpolatory quadrature formula (3.1).

(i) If $\tau_{\nu}=\tau_{\nu}^{(3)}$, then the error term $R_{n}=R_{n}^{(3)}$ satisfies

$$
(-1)^{k} R_{n}^{(3)}\left(t^{k}\right)>0 \quad \text { for all } k \geq n .
$$

(ii) If $\tau_{\nu}=\tau_{\nu}^{(4)}$, then the error term $R_{n}=R_{n}^{(4)}$ satisfies

$$
R_{n}^{(4)}\left(t^{k}\right)>0 \quad \text { for all } k \geq n .
$$


Now, in view of $\left(3.17_{e}\right)-\left(3.17_{o}\right)$ and $(3.30)-(3.31)$, representation $\left(3.4_{i}\right)$ with $R_{n}=R_{n}^{(2)}$ or $R_{n}=R_{n}^{(4)}$, by means of $(2.9)$ with $\pi_{n}=U_{n}$ or $\pi_{n}=W_{n}$, and representation $\left(3.4_{i i}\right)$ with $R_{n}=R_{n}^{(3)}$, by means of (2.11) with $\pi_{n}=V_{n}$, lead to the following.

Proposition 3.3. Consider the interpolatory quadrature formula (3.1).

(i) If $\tau_{\nu}=\tau_{\nu}^{(2)}$, then the norm of the error functional $R_{n}=R_{n}^{(2)}$ is given by

$$
\left\|R_{n}^{(2)}\right\|=r \ln \left(\frac{r+1}{r-1}\right)-\frac{4 r}{U_{n}(r)} \sum_{k=1}^{[(n+1) / 2]} \frac{U_{n-2 k+1}(r)}{2 k-1} .
$$

(ii) If $\tau_{\nu}=\tau_{\nu}^{(3)}$, then, for $1 \leq n \leq 20$, the norm of the error functional $R_{n}=R_{n}^{(3)}$ is given by

$$
\left\|R_{n}^{(3)}\right\|=r \ln \left(\frac{r+1}{r-1}\right)+\frac{4 r}{V_{n}(-r)} \sum_{k=1}^{[(n+1) / 2]} \frac{V_{n-2 k+1}(-r)}{2 k-1} .
$$

(iii) If $\tau_{\nu}=\tau_{\nu}^{(4)}$, then, for $1 \leq n \leq 20$, the norm of the error functional $R_{n}=$ $R_{n}^{(4)}$ is given by

$$
\left\|R_{n}^{(4)}\right\|=r \ln \left(\frac{r+1}{r-1}\right)-\frac{4 r}{W_{n}(r)} \sum_{k=1}^{[(n+1) / 2]} \frac{W_{n-2 k+1}(r)}{2 k-1} .
$$

In [1, Equations (1.7.5)-(1.7.6)], Akrivis has shown that

$$
\left\|R_{n}^{(2)}\right\| \leq\left\{\begin{array}{c}
\frac{8(n+1) r \tau^{n+1}}{1-\tau^{2 n+2}}\left\{\frac{1}{2(n+1)^{2}}+\frac{\tau^{2}}{n^{2}+2 n-3}+\frac{\tau^{4}}{n^{2}+2 n-15}+\frac{1}{2 n+1} \frac{\tau^{6}-\tau^{n+2}}{1-\tau^{2}}\right\} \\
\text { for } n(\text { even }) \geq 4, \\
\frac{8(n+1) r \tau^{n+2}}{1-\tau^{2 n+2}}\left\{\frac{1}{n(n+2)}+\frac{\tau^{2}}{n^{2}+2 n-8}+\frac{\tau^{4}}{n^{2}+2 n-24}+\frac{1}{2 n+1} \frac{\tau^{6}-\tau^{n+1}}{1-\tau^{2}}\right\} \\
\text { for } n(\text { odd }) \geq 5 \\
\left\|R_{1}^{(2)}\right\| \leq \frac{16 r \tau^{3}}{3\left(1-\tau^{4}\right)}, \\
\left\|R_{2}^{(2)}\right\| \leq \frac{24 r \tau^{3}}{1-\tau^{6}}\left(\frac{1}{18}+\frac{\tau^{2}}{5}\right), \\
\left\|R_{3}^{(2)}\right\| \leq \frac{32 r \tau^{5}}{1-\tau^{8}}\left(\frac{1}{15}+\frac{\tau^{2}}{7}\right),
\end{array}\right.
$$

where $\tau=r-\sqrt{r^{2}-1}$. This bound for $\left\|R_{n}^{(2)}\right\|$ is very close to its exact value, given in (3.32), so, in that sense, our result for $\left\|R_{n}^{(2)}\right\|$ is a refinement of Akrivis's result.

The explicit forms (3.32)-(3.34) for the norm of the error functionals lead to bounds of type (3.6) and (3.12) for the error term of formula (3.1) with the $\tau_{\nu}$ given by (3.13)-(3.15). The quality of these bounds is illustrated in the following section.

Remark 1. Besides formula (3.1) with the $\tau_{\nu}$ given by (3.13)-(3.15), there are no other interpolatory formulae with Chebyshev abscissae, including the well-known Fejér rule of the first kind, or Pólya rule, and the Clenshaw-Curtis rule, whose error term satisfies either $\left(3.3_{i}\right)$ or $\left(3.3_{i i}\right)$, which are essential in deriving representations $\left(3.4_{i}\right)$ and $\left(3.4_{i i}\right)$. 


\section{A nUmerical EXAMPle}

We want to approximate the integral

$$
\int_{-1}^{1} e^{\omega t} d t=\frac{e^{\omega}-e^{-\omega}}{\omega}, \omega>0
$$

by using formula (3.1) with $\tau_{\nu}=\tau_{\nu}^{(2)}$ or $\tau_{\nu}=\tau_{\nu}^{(3)}$.

The function $f(z)=e^{\omega z}=\sum_{k=0}^{\infty} \frac{\omega^{k} z^{k}}{k !}$ is entire and, in view of (3.2) and $\left(3.17_{o}\right)$, $(4.2)$

$$
|f|_{r}^{(2)}= \begin{cases}\frac{\omega^{2[(n+1) / 2]} r^{2[(n+1) / 2]}}{(2[(n+1) / 2]) !}, & 1<r \leq \frac{\sqrt{(2[(n+1) / 2]+1)(2[(n+1) / 2]+2)}}{\omega}, \\ \frac{\omega^{2([(n+1) / 2]+k)} r^{2([(n+1) / 2]+k)}}{(2([(n+1) / 2]+k)) !}, & \frac{\sqrt{(2[(n+1) / 2]+2 k-1)(2[(n+1) / 2]+2 k)}}{\omega}<r \\ & \leq \frac{\sqrt{(2[(n+1) / 2]+2 k+1)(2[(n+1) / 2]+2 k+2)}}{\omega}, k=1,2, \ldots,\end{cases}
$$

with [.] denoting the integer part of a real number. The above formula holds as it stands if

$$
\sqrt{(2[(n+1) / 2]+1)(2[(n+1) / 2]+2)}>\omega ;
$$

in case that

$$
\sqrt{(2[(n+1) / 2]+1)(2[(n+1) / 2]+2)} \leq \omega,
$$

the formula for $|f|_{r}^{(2)}$ starts at the branch of (4.2) for which

$$
\sqrt{(2[(n+1) / 2]+2 k+1)(2[(n+1) / 2]+2 k+2)}>\omega .
$$

Similarly,

$$
|f|_{r}^{(3)}= \begin{cases}\frac{\omega^{n} r^{n}}{n !}, & 1<r \leq \frac{n+1}{\omega} \\ \frac{\omega^{n+k} r^{n+k}}{(n+k) !}, & \frac{n+k}{\omega}<r \leq \frac{n+k+1}{\omega}, \quad k=1,2, \ldots,\end{cases}
$$

assuming that $n+1>\omega$; otherwise, the formula for $|f|_{r}^{(3)}$ starts at the branch of (4.3) for which $n+k+1>\omega$. Therefore, in both cases, $f \in X_{\infty}$. In addition,

$$
\max _{|z|=r}|f(z)|=e^{\omega r} \text {. }
$$

Hence, the error functionals $R_{n}^{(2)}(f)$ and $R_{n}^{(3)}(f)$ can be estimated by means of (3.6) and (3.12), i.e.,

$$
\begin{aligned}
& \left|R_{n}^{(2)}(f)\right| \leq \inf _{1<r<\infty}\left(\left\|R_{n}^{(2)}\right\||f|_{r}^{(2)}\right), \\
& \left|R_{n}^{(3)}(f)\right| \leq \inf _{1<r<\infty}\left(\left\|R_{n}^{(3)}\right\||f|_{r}^{(3)}\right),
\end{aligned}
$$

and

$$
\begin{aligned}
& \left|R_{n}^{(2)}(f)\right| \leq \inf _{1<r<\infty}\left(\left\|R_{n}^{(2)}\right\| e^{\omega r}\right), \\
& \left|R_{n}^{(3)}(f)\right| \leq \inf _{1<r<\infty}\left(\left\|R_{n}^{(3)}\right\| e^{\omega r}\right),
\end{aligned}
$$

with the $\left\|R_{n}^{(2)}\right\|$ and $\left\|R_{n}^{(3)}\right\|$ given by (3.32) and (3.33). 
Also, it is interesting to see how estimates (4.4) and (4.6) compare with already existing ones. We chose two such estimates. The first is a traditional one, obtained from $\left(3.16_{e}\right)$ and $\left(3.16_{o}\right)$,

$$
\left|R_{n}^{(2)}(f)\right| \leq \begin{cases}\frac{\omega^{n} e^{\omega}}{2^{n-1}(n+1) !} & \text { if } n \text { is even, } \\ \frac{(n+1) \omega^{n+1} e^{\omega}}{2^{n-1} n(n+2) !} & \text { if } n \text { is odd. }\end{cases}
$$

The second estimate, given by Basu in [3], was obtained by the contour integration method of the previous section applied on the ellipse

$$
\mathcal{E}_{\rho}=\left\{z \in \mathbb{C}: z=\frac{1}{2}\left(u+u^{-1}\right), \quad u=\rho e^{i \theta}, \quad 0 \leq \theta \leq 2 \pi\right\}
$$

with foci at $z= \pm 1$ and sum of semiaxes equal to $\rho, \rho>1$,

$$
\left|R_{n}^{(2)}(f)\right| \leq\left\{\frac{\sigma_{n, n+3}}{\rho^{2}-1}+\frac{1}{\rho^{n+1}} \frac{4(n+1)}{2 n+3}\right\} \frac{\left(\rho+\rho^{-1}\right)^{2}}{\rho^{n+1}-\rho^{-(n+1)}} \max _{z \in \mathcal{E}_{\rho}}|f(z)|, \quad n \text { odd },
$$

where

$$
\sigma_{n, n+3}=2 \sum_{k=1}^{n+1} \frac{1}{2 k+1}
$$

Since in our case

$$
\max _{z \in \mathcal{E}_{\rho}}|f(z)|=e^{\frac{1}{2} \omega\left(\rho+\rho^{-1}\right)}
$$

we get

$$
\left|R_{n}^{(2)}(f)\right| \leq\left\{\frac{\sigma_{n, n+3}}{\rho^{2}-1}+\frac{1}{\rho^{n+1}} \frac{4(n+1)}{2 n+3}\right\} \frac{\left(\rho+\rho^{-1}\right)^{2}}{\rho^{n+1}-\rho^{-(n+1)}} e^{\frac{1}{2} \omega\left(\rho+\rho^{-1}\right)}, \quad n \text { odd, }
$$

which can be optimized as a function of $\rho$ giving

$$
\left|R_{n}^{(2)}(f)\right| \leq \inf _{1<\rho<\infty}\left(\left\{\frac{\sigma_{n, n+3}}{\rho^{2}-1}+\frac{1}{\rho^{n+1}} \frac{4(n+1)}{2 n+3}\right\} \frac{\left(\rho+\rho^{-1}\right)^{2}}{\rho^{n+1}-\rho^{-(n+1)}} e^{\frac{1}{2} \omega\left(\rho+\rho^{-1}\right)}\right), \quad n \text { odd } .
$$

Our results are summarized in Tables 4.1 and 4.2 for the case $\tau_{\nu}=\tau_{\nu}^{(2)}$ and in Table 4.3 for the case $\tau_{\nu}=\tau_{\nu}^{(3)}$. (Numbers in parentheses indicate decimal exponents.) All computations were performed on a SUN Ultra 5 computer in quad precision (machine precision $1.93 \cdot 10^{-34}$ ). The value of $r$ or $\rho$, at which the infimum in each of bounds (4.4)-(4.7) and (4.9) was attained, is given in the column headed $r_{\text {opt }}$ and $\rho_{\text {opt }}$, respectively, which is placed immediately before the column of the corresponding bound. As $n$ and $r$ increase, $\left\|R_{n}^{(2)}\right\|$ and $\left\|R_{n}^{(3)}\right\|$ decrease and close to machine precision they can even take a negative value. This actually happens, for $\tau_{\nu}=\tau_{\nu}^{(2)}$ when $\omega=0.5, n \geq 15$ and $\omega=1.0, n \geq 19$, and for $\tau_{\nu}=\tau_{\nu}^{(3)}$ when $\omega=0.5, n \geq 16$ and $\omega=1.0, n \geq 19$. The reason is that, in all these cases, the infimums in bounds (4.4)-(4.7) are attained at values of $r>20$.

Bounds (4.4)-(4.5) provide an excellent estimate of the actual error, and are always somewhat better than bounds (4.6)-(4.7), respectively. This is to be expected, since (4.6)-(4.7) can be derived from (4.4)-(4.5) if $|f|_{r}^{(2)}$ and $|f|_{r}^{(3)}$ are estimated by $\max _{|z|=r}|f(z)|$. In addition, bounds (4.4) and (4.6) are consistently better than bounds (4.8) and (4.9). Bound (4.8), for formula (3.1) with $\tau_{\nu}=\tau_{\nu}^{(2)}$, is the best among the error bounds that use higher order derivatives of the function $f$. Bound 
TABLE 4.1. Error bounds and actual error in approximating the integral (4.1) using formula (3.1) with $\tau_{\nu}=\tau_{\nu}^{(2)}$.

\begin{tabular}{ccccccc}
\hline$\omega$ & $n$ & $r_{\text {opt }}$ & Bound (4.4) & $r_{\text {opt }}$ & Bound (4.6) & Error \\
\hline 0.5 & 5 & 14.966 & $2.347(-7)$ & 12.057 & $1.469(-6)$ & $2.347(-7)$ \\
& 10 & 22.978 & $4.808(-14)$ & 20.033 & $3.851(-13)$ & $4.808(-14)$ \\
1.0 & 5 & 7.483 & $1.545(-5)$ & 6.114 & $9.810(-5)$ & $1.544(-5)$ \\
& 10 & 11.489 & $5.016(-11)$ & 10.065 & $4.040(-10)$ & $5.015(-11)$ \\
& 15 & 17.492 & $1.858(-19)$ & 16.035 & $1.878(-18)$ & $1.858(-19)$ \\
2.0 & 5 & 3.741 & $1.110(-3)$ & 3.220 & $7.425(-3)$ & $1.103(-3)$ \\
& 10 & 5.744 & $5.539(-8)$ & 5.128 & $4.558(-7)$ & $5.529(-8)$ \\
& 15 & 8.746 & $1.273(-14)$ & 8.070 & $1.297(-13)$ & $1.272(-14)$ \\
& 20 & 10.747 & $4.115(-20)$ & 10.057 & $4.667(-19)$ & $4.114(-20)$ \\
4.0 & 5 & 1.871 & $1.210(-1)$ & 1.889 & $8.818(-1)$ & $1.084(-1)$ \\
& 10 & 2.872 & $7.783(-5)$ & 2.743 & $6.785(-4)$ & $7.568(-5)$ \\
& 15 & 4.373 & $1.000(-9)$ & 4.138 & $1.047(-8)$ & $9.936(-10)$ \\
& 20 & 5.373 & $5.024(-14)$ & 5.113 & $5.807(-13)$ & $5.005(-14)$ \\
8.0 & 5 & 1.383 & $4.984(+1)$ & 1.329 & $4.111(+2)$ & $3.266(+1)$ \\
& 10 & 1.686 & $2.806(-1)$ & 1.668 & $2.668(0)$ & $2.309(-1)$ \\
& 15 & 2.302 & $1.434(-4)$ & 2.261 & $1.534(-3)$ & $1.292(-4)$ \\
& 20 & 2.687 & $1.001(-7)$ & 2.718 & $1.186(-6)$ & $9.407(-8)$ \\
\hline
\end{tabular}

TABLE 4.2. Error bounds and actual error in approximating the integral (4.1) using formula (3.1) with $\tau_{\nu}=\tau_{\nu}^{(2)}$.

\begin{tabular}{cccccc}
\hline$\omega$ & $n$ & Bound (4.8) & $\rho_{\text {opt }}$ & Bound (4.9) & Error \\
\hline 0.5 & 5 & $3.834(-7)$ & 24.081 & $4.096(-6)$ & $2.347(-7)$ \\
& 10 & $7.878(-14)$ & - & - & $4.808(-14)$ \\
1.0 & 5 & $4.045(-5)$ & 12.163 & $2.746(-4)$ & $1.544(-5)$ \\
& 10 & $1.330(-10)$ & - & - & $5.015(-11)$ \\
& 15 & $4.975(-19)$ & 32.042 & $2.094(-17)$ & $1.858(-19)$ \\
2.0 & 5 & $7.037(-3)$ & 6.310 & $2.104(-2)$ & $1.103(-3)$ \\
& 10 & $3.702(-7)$ & - & - & $5.529(-8)$ \\
& 15 & $8.864(-14)$ & 16.085 & $1.451(-12)$ & $1.272(-14)$ \\
& 20 & $2.893(-19)$ & - & - & $4.114(-20)$ \\
4.0 & 5 & $3.328(0)$ & 3.530 & $2.585(0)$ & $1.084(-1)$ \\
& 10 & $2.801(-3)$ & - & - & $7.568(-5)$ \\
& 15 & $4.292(-8)$ & 8.167 & $1.184(-7)$ & $9.936(-10)$ \\
& 20 & $2.241(-12)$ & - & - & $5.005(-14)$ \\
8.0 & 5 & $1.163(+4)$ & 2.256 & $1.300(+3)$ & $3.266(+1)$ \\
& 10 & $1.566(+2)$ & - & - & $2.309(-1)$ \\
& 15 & $1.536(-1)$ & 4.311 & $1.798(-2)$ & $1.292(-4)$ \\
& 20 & $1.283(-4)$ & - & - & $9.407(-8)$ \\
\hline & & & & &
\end{tabular}

(4.9) was obtained by the same method as that used in order to derive bound (4.6), except that in the case of (4.9) the method was applied on an elliptic, instead of a circular, contour. Ellipses have the advantage of shrinking around the interval $[-1,1]$ as the sum of the semiaxes $\rho \rightarrow 1$, and that way give better results than 
TABLE 4.3. Error bounds and actual error in approximating the integral (4.1) using formula (3.1) with $\tau_{\nu}=\tau_{\nu}^{(3)}$.

\begin{tabular}{ccccccc}
\hline$\omega$ & $n$ & $r_{\text {opt }}$ & Bound (4.5) & $r_{\text {opt }}$ & Bound (4.7) & Error \\
\hline 0.5 & 5 & 12.000 & $3.397(-6)$ & 10.143 & $1.958(-5)$ & $3.195(-6)$ \\
& 10 & 22.000 & $4.941(-14)$ & 20.089 & $3.965(-13)$ & $4.672(-14)$ \\
& 15 & 31.990 & $9.667(-23)$ & 30.042 & $9.451(-22)$ & $9.402(-23)$ \\
1.0 & 5 & 6.000 & $1.165(-4)$ & 5.211 & $6.847(-4)$ & $1.026(-4)$ \\
& 10 & 11.000 & $5.294(-11)$ & 10.116 & $4.273(-10)$ & $4.726(-11)$ \\
& 15 & 16.000 & $3.252(-18)$ & 15.066 & $3.187(-17)$ & $3.074(-18)$ \\
2.0 & 5 & 3.000 & $4.676(-3)$ & 2.829 & $2.876(-2)$ & $3.539(-3)$ \\
& 10 & 5.500 & $6.155(-8)$ & 5.169 & $5.047(-7)$ & $4.873(-8)$ \\
& 15 & 8.000 & $1.151(-13)$ & 7.603 & $1.137(-12)$ & $1.026(-13)$ \\
& 20 & 10.500 & $4.332(-20)$ & 10.083 & $4.911(-19)$ & $3.889(-20)$ \\
4.0 & 5 & 1.750 & $3.172(-1)$ & 1.745 & $2.129(0)$ & $1.719(-1)$ \\
& 10 & 2.865 & $9.557(-5)$ & 2.768 & $8.017(-4)$ & $5.713(-5)$ \\
& 15 & 4.000 & $4.889(-9)$ & 3.923 & $4.912(-8)$ & $3.827(-9)$ \\
& 20 & 5.250 & $5.556(-14)$ & 5.132 & $6.372(-13)$ & $4.440(-14)$ \\
8.0 & 5 & 1.295 & $8.876(+1)$ & 1.280 & $7.125(+2)$ & $3.056(+1)$ \\
& 10 & 1.750 & $3.616(-1)$ & 1.675 & $3.339(0)$ & $1.192(-1)$ \\
& 15 & 2.250 & $3.946(-4)$ & 2.172 & $4.157(-3)$ & $2.319(-4)$ \\
& 20 & 2.750 & $1.171(-7)$ & 2.729 & $1.381(-6)$ & $7.218(-8)$ \\
\hline
\end{tabular}

circles. However, bound (4.9) uses an estimate for the kernel $K_{n}$ (cf. (3.7)-(3.8)), which is poor for $\rho$ close to 1 and reasonably good for large $\rho$ (when the ellipse looks more and more like a circle), and this apparently accounts for (4.9) giving results substantially less sharp than bound (4.6).

\section{REFERENCES}

[1] G. AKRIVIS, Fehlerabschätzungen bei der numerischen Integration in einer und mehreren Dimensionen, Doctoral Dissertation, Ludwig-Maximilians-Universität München, 1982.

[2] R.G. Bartle, The Elements of Real Analysis, 2nd ed., John Wiley \& Sons, New York, 1976. MR0393369 (52:14179)

[3] N.K. BASU, "Error estimates for a Chebyshev quadrature method", Math. Comp., v. 24, 1970, pp. 863-867. MR0277111 (43:2848)

[4] P.J. Davis And P. Rabinowitz, Methods of Numerical Integration, 2nd ed., Academic Press, San Diego, 1984. MR0760629 (86d:65004)

[5] W. Gautschi, "Remainder estimates for analytic functions" in (T.O. Espelid and A. Genz, eds.), Numerical Integration, Kluwer Academic Publishers, Dordrecht/Boston/London, 1992, pp. 133-145. MR.1198903 (94e:41049)

[6] G. HämmerLin, "Fehlerabschätzung bei numerischer Integration nach Gauss" in (B. Brosowski and E. Martensen, eds.), Methoden und Verfahren der mathematischen Physik, vol. 6, Bibliographisches Institut, Mannheim-Wien-Zürich, 1972, pp. 153-163. MR0359277 $(50: 11732)$

[7] S.E. NOTARIS, "Interpolatory quadrature formulae with Chebyshev abscissae", J. Comput. Appl. Math., v. 133, 2001, pp. 507-517. MR1858308 (2002g:65027)

Department of Mathematics, University of Athens, Panepistemiopolis, 15784 ZograFOU, GREECE

E-mail address: notaris@math.uoa.gr 